\title{
On a mixed Littlewood conjecture for quadratic numbers
}

\author{
par BERNARD DE MATHAN
}

\begin{abstract}
RÉSUMÉ. Nous étudions un problème diophantien simultané relié à la conjecture de Littlewood. En utilisant des minorations connues de formes linéaires de logarithmes $p$-adiques, nous montrons qu'un résultat que nous avons précédemment obtenu, concernant les nombres quadratiques, est presque optimal.
\end{abstract}

ABSTRACT. We study a simultaneous diophantine problem related to Littlewood's conjecture. Using known estimates for linear forms in $p$-adic logarithms, we prove that a previous result, concerning the particular case of quadratic numbers, is close to be the best possible.

\section{Introduction}

In a joint paper, with $O$. Teulié [5], we have considered the following problem. Let $\mathcal{B}=\left(b_{k}\right)_{k \geq 1}$ be a sequence of integers greater than 1 . Consider the sequence $\left(r_{n}\right)_{n \geq 0}$, where $r_{0}=1$ and $r_{n}=\prod_{0<k \leq n} b_{k}$ for $n>0$. For $q \in \mathbb{Z}$, set

$$
w_{\mathcal{B}}(q)=\sup \left\{n \in \mathbb{N} ; q \in r_{n} \mathbb{Z}\right\}
$$

and

$$
|q|_{\mathcal{B}}=\inf \left\{1 / r_{n} ; q \in r_{n} \mathbb{Z}\right\} .
$$

Notice that $|\cdot|_{\mathcal{B}}$ is not necessarily an absolute value, but when $\mathcal{B}$ is the constant sequence $p$, where $p$ is a prime number, then $|\cdot|_{\mathcal{B}}$ is the usual $p$-adic value.

For $x \in \mathbb{R}$, we denote by $\{x\}$ the number in $[-1 / 2,1 / 2[$ such that $x-\{x\} \in \mathbb{Z}$. As usual, we put $\|x\|=|\{x\}|$.

Let $\alpha$ be a real number. Given a positive integer $M$, Dirichlet's Theorem asserts that for any $n$, there exists an integer $q$, with $0<q \leq M r_{n}$, satisfying simultaneously the approximation condition $\|q \alpha\|<1 / M$ and the divisibility condition $r_{n} \mid q$, i. e. $|q|_{\mathcal{B}} \leq 1 / r_{n}$. Indeed, it is enough to 
apply Dirichlet's Theorem to the number $r_{n} \alpha$. We thus find positive integers $q$ with

$$
q\|q \alpha\||q|_{\mathcal{B}}<1 .
$$

By analogy with Littlewood's conjecture, we ask whether

$$
\inf _{q \in \mathbb{N}^{*}} q\|q \alpha\||q|_{\mathcal{B}}=0
$$

holds. The problem is trivial for $\alpha$ rational, and for an irrational number $\alpha$, one can easily see [5] that condition (1) is equivalent to the following: for each $n \in \mathbb{N}$, consider the continued fraction expansion

$$
r_{n} \alpha=\left[a_{0, n} ; a_{1, n}, \ldots, a_{k, n} \ldots\right] .
$$

We have (1) if and only if

$$
\sup _{n \geq 0, k \geq 1} a_{k, n}=+\infty
$$

However, we shall not use this characterization here.

We do not know whether (1) is satisfied for any real number $\alpha$. In [5], we have proved that if we assume that the sequence $\mathcal{B}=\left(b_{k}\right)_{k \geq 1}$ is bounded, (1) is true for every quadratic number $\alpha$. More precisely:

Theorem 1.1. (de Mathan and Teulié [5]) Suppose that the sequence $\mathcal{B}$ is bounded. Let $\alpha$ be a quadratic real number. Then there exists an infinite set of integers $q>1$ with

$$
\|q \alpha\| \ll 1 / q
$$

and

$$
|q|_{\mathcal{B}} \ll 1 / \ln q
$$

In particular, we have

$$
\liminf _{q \longrightarrow+\infty} q \ln q\|q \alpha\||q|_{\mathcal{B}}<+\infty .
$$

As usual, for positive functions $x$ and $y$, the notation $x \ll y$ means that there exists a positive constant $C$ such that $x \leq C y$.

In our lecture at Graz, for the "Journées Arithmétiques 2003", it was discussed whether the factor $\ln q$ in (3) is best possible. We do not know the answer to this question, but we shall prove: 
Theorem 1.2. Assume that the sequence $\mathcal{B}$ is bounded. Let $\alpha$ be a real quadratic number, and let $\mathcal{S}$ be a set of integers $q>1$ with

$$
\|q \alpha\| \ll 1 / q \text {. }
$$

Then there exists a constant $\lambda=\lambda(\mathcal{S})$ such that

$$
|q|_{\mathcal{B}} \gg \frac{1}{(\ln q)^{\lambda}}
$$

for any $q \in \mathcal{S}$.

One may expect that (4) holds for any $\lambda>1$, but we are not able to prove this. We do not even know whether there exists a real number $\lambda$ for which (4) holds for any set $\mathcal{S}$ of integers $q>1$ satisfying (2). Indeed, Theorem 1.2 does not ensure that $\sup _{\mathcal{S}} \lambda(\mathcal{S})<+\infty$.

There is some analogy between this problem, and the classical simultaneous Diophantine approximation. For instance, let us recall Peck's Theorem. Let $n$ be an integer greater than 1 , and let $\alpha_{1}, \ldots, \alpha_{n}$, be $n$ numbers in a real algebraic number field of degree $n+1$ over $\mathbb{Q}$. Then it was proved by Peck [7] that there exists an infinite set of integers $q>1$ with

$$
\left\|q \alpha_{k}\right\| \ll(\ln q)^{-1 /(n-1)} q^{-1 / n}
$$

for $1 \leq k<n$, and

$$
\left\|q \alpha_{n}\right\| \ll q^{-1 / n} .
$$

Assume that $1, \alpha_{1}, \ldots, \alpha_{n}$ are linearly independent over $\mathbb{Q}$, and let $\mathcal{S}$ be an infinite set of integers $q>1$, with

$$
\left\|q \alpha_{k}\right\| \ll q^{-1 / n}
$$

for each $1 \leq k \leq n$. Then we have proved in [3] that there exists a constant $\kappa=\kappa(\mathcal{S})$ such that

$$
\max _{1 \leq k<n}\left\|q \alpha_{k}\right\| \gg(\ln q)^{-\kappa} q^{-1 / n} .
$$

Theorem 1.2 can be regarded as an analogue of this result with $n=1$, and its proof is similar.

\section{Proof of the result}

\subsection{Some rational approximations of $\alpha$.}

In the quadratic field $\mathbb{Q}(\alpha)$, there exists a unit $\omega$ of infinite order. Replacing, if necessary, $\omega$ by $\omega^{2}$ or $1 / \omega^{2}$, we may suppose $\omega>1$. In his original work, Peck uses units which are "large" and whose other conjugates are "small" and close to be equal. Here, Peck's units are just the $\omega^{m}$ 's, with $m \in \mathbb{N}$. We shall use these units in order to describe the rational approximations of $\alpha$ which satisfy (2). 
Denote by $\sigma_{0}=$ id and $\sigma_{1}=\sigma$ the automorphisms of $\mathbb{Q}(\alpha)$. As usual, we denote by $\operatorname{Tr}$ the trace form $\operatorname{Tr}_{\mathbb{Q}(\alpha) / \mathbb{Q}}=\sigma_{0}+\sigma_{1}$. The basis $(1, \alpha)$ of $\mathbb{Q}(\alpha)$ admits a dual basis $\left(\beta_{0}, \beta_{1}\right)$ for the non-degenerate $\mathbb{Q}$-bilinear form $(x, y) \longmapsto \operatorname{Tr}(x y)$ on $\mathbb{Q}(\alpha)$. That means that, if we set $\alpha_{0}=1$ and $\alpha_{1}=\alpha$, we have $\operatorname{Tr}\left(\alpha_{k} \beta_{l}\right)=\delta_{k l}$, for $k=0,1$ and $l=0,1$, where $\delta_{l l}=1$, and $\delta_{k l}=0$ if $k \neq l$. Here it is easy to calculate $\beta_{0}=-\frac{\sigma(\alpha)}{\alpha-\sigma(\alpha)}$ and $\beta_{1}=\frac{1}{\alpha-\sigma(\alpha)}$. Hence, if we put

$$
\eta=\frac{-q \sigma(\alpha)+q^{\prime}}{\alpha-\sigma(\alpha)}
$$

where $q$ and $q^{\prime}$ are rational numbers, we have

$$
q=\operatorname{Tr} \eta
$$

and

$$
q^{\prime}=\operatorname{Tr}(\alpha \eta)
$$

Also notice that (5) and (6) imply that

$$
q \alpha-q^{\prime}=(\alpha-\sigma(\alpha)) \sigma(\eta) .
$$

Let $D$ be a positive integer such that $D \alpha, \frac{D}{\alpha-\sigma(\alpha)}$, and $\frac{D \alpha}{\alpha-\sigma(\alpha)}$ are algebraic integers.

The notation $A \asymp B$, where $A$ and $B$ are positive quantities, means that $B \ll A \ll B$.

Lemma 2.1. Let $\gamma$ be a positive number in $\mathbb{Q}(\alpha)$. Let $\Delta$ be a positive integer such that $\Delta \gamma$ is an algebraic integer. For each $m \in \mathbb{N}$, define the rational number

$$
q=q(m)=\operatorname{Tr}\left(\gamma \omega^{m}\right) .
$$

Then $\Delta q$ is a rational integer, one has $q>0$ when $m$ is large, and the integers $D \Delta q$ satisfy (2).

Proof. Also define

$$
q^{\prime}=q^{\prime}(m)=\operatorname{Tr}\left(\alpha \gamma \omega^{m}\right) .
$$

As $\Delta \gamma \omega^{m}$ and $D \Delta \alpha \gamma \omega^{m}$ are algebraic integers, $\Delta q$ and $D \Delta q^{\prime}$ are rational integers. As $\sigma(\omega)=1 / \omega$, we have $q=\gamma \omega^{m}+\sigma(\gamma) \omega^{-m}$, hence $q>0$ as soon as $\omega^{2 m}>-\sigma(\gamma) / \gamma$, and then

$$
q \asymp \omega^{m} \text {. }
$$

From (7), we get $q \alpha-q^{\prime}=(\alpha-\sigma(\alpha)) \sigma(\gamma) \omega^{-m}$, hence

$$
\left|q \alpha-q^{\prime}\right| \asymp \omega^{-m} \text {. }
$$

As $D \Delta q$ and $D \Delta q^{\prime}$ are integers, it follows from (10) that for large $m$ we have $\|D \Delta q \alpha\|=D \Delta\left|q \alpha-q^{\prime}\right|$, and by (9) and (10), the integers $D \Delta q$ satisfy (2). 
Conversely:

Lemma 2.2. Let $\mathcal{S}$ be a set of positive integers $q$ satisfying (2) . Then there exists a finite set $\Gamma$ of numbers $\gamma \in \mathbb{Q}(\alpha), \gamma \neq 0$, such that for any $q \in \mathcal{S}$, there exist $\gamma \in \Gamma$ and $m \in \mathbb{N}$ such that

$$
q=\operatorname{Tr}\left(\gamma \omega^{m}\right) \text {. }
$$

Proof. For $q \in \mathcal{S}$, let $m(q)=m$ be the positive integer such that $\omega^{m-1} \leq q<\omega^{m}$. We thus have $\omega^{m} \asymp q$. Let $q^{\prime}$ be the rational integer such that $\{q \alpha\}=q \alpha-q^{\prime}$. Set

$$
\gamma=\frac{-q \sigma(\alpha)+q^{\prime}}{\alpha-\sigma(\alpha)} \omega^{-m}
$$

First, notice that $D \gamma$ is an algebraic integer. From (5), we get (8). Writing

$$
\gamma \omega^{m}=q-\frac{q \alpha-q^{\prime}}{\alpha-\sigma(\alpha)}
$$

we see that $\gamma>0$ when $q$ is large, and $\gamma \omega^{m} \asymp q$. As we have $\omega^{m} \asymp q$, we thus get $\gamma \asymp 1$. We also have

$$
\sigma(\gamma)=\frac{q \alpha-q^{\prime}}{\alpha-\sigma(\alpha)} \omega^{m}
$$

hence, by (2), $|\sigma(\gamma)| \ll \omega^{m} / q$, and thus, $|\sigma(\gamma)| \ll 1$. Then, as $D \gamma$ is an algebraic integer in $\mathbb{Q}(\alpha)$, and $\max (|\gamma|,|\sigma(\gamma)|) \ll 1$, the set of the $\gamma$ 's is finite.

\subsection{End of proof.}

Denote by $P$ the set of all prime numbers dividing one of the $b_{k}$. Since we assume that the sequence $\left(b_{k}\right)$ is bounded, this set is finite. For $p \in P$, we extend the $p$-adic absolute value to $\mathbb{Q}(\alpha)$. The completion of this field is $\mathbb{Q}_{p}(\alpha)$. As above, let $\omega$ be a unit in $\mathbb{Q}(\alpha)$ with $\omega>1$. Note that $|\omega|_{p}=1$. The ball $\left\{x \in \mathbb{Q}_{p}(\alpha) ;|x-1|_{p}<p^{-1 /(p-1)}\right\}$ is a subgroup of finite index in the multiplicative group $\left\{x \in \mathbb{Q}_{p}(\alpha) ;|x|_{p}=1\right\}$. Hence, replacing $\omega$ by $\omega^{n}$, where $n$ is a suitable positive integer, we may also suppose that $|\omega-1|_{p}<p^{-1 /(p-1)}$ for every $p \in P$.

We shall use the $p$-adic logarithm function, which is defined on the multiplicative group $\left\{x \in \mathbb{C}_{p} ;|x-1|_{p}<1\right\} \subset \mathbb{C}_{p}$ by

$$
\log x=\sum_{n=1}^{+\infty}(-1)^{n-1} \frac{(x-1)^{n}}{n} .
$$

This function satisfies

$$
\log x y=\log x+\log y
$$


and, for $|x-1|_{p}<p^{-1 /(p-1)},|\log x|_{p}=|x-1|_{p}$. Hence, for $|x-1|_{p}<$ $p^{-1 /(p-1)}$ and $|y-1|_{p}<p^{-1 /(p-1)}$, we have

$$
|\log x-\log y|_{p}=\left|\log \frac{x}{y}\right|_{p}=\left|\frac{x}{y}-1\right|_{p}=|x-y|_{p} .
$$

We prove:

Lemma 2.3. Let $p$ be a number of $P$. Let $\gamma$ be a positive number of $\mathbb{Q}(\alpha)$. For $m \in \mathbb{N}$, set

$$
q=q(m)=\operatorname{Tr}\left(\gamma \omega^{m}\right) .
$$

Then, if

$$
\left|\frac{\sigma(\gamma)}{\gamma}+1\right|_{p} \geq p^{-1 /(p-1)}
$$

we have

$$
|q|_{p} \asymp 1
$$

for large $m$; if

$$
\left|\frac{\sigma(\gamma)}{\gamma}+1\right|_{p}<p^{-1 /(p-1)}
$$

then

$$
|q|_{p} \asymp|2 m \log \omega-\log (-\sigma(\gamma) / \gamma)|_{p} .
$$

Proof. Recall that $q>0$ when $m$ is large (Lemma 2.1). From the definition, we get for each $p \in P,|q|_{p}=\left|\gamma \omega^{m}+\sigma(\gamma) \omega^{-m}\right|_{p}=|\gamma|_{p}\left|\omega^{2 m}-\delta\right|_{p}$, where $\delta=-\sigma(\gamma) / \gamma$. If $|\delta-1|_{p} \geq p^{-1 /(p-1)}$, we have $\left|\omega^{2 m}-\delta\right|_{p} \geq p^{-1 /(p-1)}$, since $|\omega-1|_{p}<p^{-1 /(p-1)}$ and $\left|\omega^{2 m}-1\right|_{p}<p^{-1 /(p-1)}$. Then we get

$$
|q|_{p} \asymp 1 \text {. }
$$

If $|\delta-1|_{p}<p^{-1 /(p-1)}$, then, by (11), we write $\left|\omega^{2 m}-\delta\right|_{p}=|2 m \log \omega-\log \delta|_{p}$, and we obtain (12).

Accordingly, in order to achieve the proof of the result, we shall use known lower bounds for linear forms in $p$-adic logarithms. For instance, it follows from [8] that:

Lemma 2.4. (K. Yu [8]) Let $x$ and $y$ be algebraic numbers in $\mathbb{C}_{p}$, with $|x-1|_{p}<p^{-1 /(p-1)}$ and $|y-1|_{p}<p^{-1 /(p-1)}$. Then there exists a real constant $\kappa$ such that for any pair $(k, \ell)$ of rational integers with $k \log x+\ell \log y \neq 0$, one has

$$
|k \log x+\ell \log y|_{p} \gg(\max (|k|,|\ell|))^{-\kappa} .
$$

Note that this result is trivial, with $\kappa=1$, if $\log x$ and $\log y$ are not linearly independent over $\mathbb{Q}$, and $\log x \neq 0$, i.e, $x \neq 1$. Indeed, if $a \log x=$ $b \log y$, where $a$ and $b$ are rational integers with $b \neq 0$, then we write $|k \log x+\ell \log y|_{p}=\frac{1}{|b|_{p}}|b k+a \ell|_{p}|x-1|_{p}$. Hence we get $|k \log x+\ell \log y|_{p} \gg$ $|b k+a \ell|_{p} \geq|b k+a \ell|^{-1} \gg\left(\max (|k|,|\ell|)^{-1}\right.$, when $k \log x+\ell \log y \neq 0$. 
We can then achieve the proof of Theorem 1.2. Applying Lemma 2.2, we can suppose that the set $\Gamma$ contains a unique element $\gamma>0$, i.e., for any $q \in \mathcal{S}$, there exists $m \in \mathbb{N}$ such that we have (8). It follows from Lemma 2.3 and 2.4 that there exists a constant $\kappa$ such that $|q|_{p} \gg m^{-\kappa}$ (one may take $\kappa=0$ if $\left.\left|\frac{\sigma(\gamma)}{\gamma}+1\right|_{p} \geq p^{-1 /(p-1)}\right)$. As $q \asymp \omega^{m}$, hence $m \asymp \ln q$, we get $|q|_{p} \gg(\ln q)^{-\kappa}$. Now set $\kappa=\kappa_{p}$ (the constant $\kappa_{p}$ may depend upon $p \in P)$. Note that $|q|_{\mathcal{B}} \geq \prod_{p \in P}|q|_{p}$. Indeed, putting $|q|_{\mathcal{B}}=1 / r_{n}$, we have $q \in r_{n} \mathbb{Z}$, hence $|q|_{p} \leq\left|r_{n}\right|_{p}$ and $\prod_{p \in P}|q|_{p} \leq \prod_{p \in P}\left|r_{n}\right|_{p}=1 / r_{n}$. We thus get (4) with $\lambda=\sum_{p \in P} \kappa_{p}$, and Theorem 1.2 is proved.

\subsection{A remark.}

Note that one may also use Lemma 2.3 for solving the opposite problem. For simplicity, consider the case where ||$_{\mathcal{B}}$ is the $p$-adic value for a prime number $p$. If we take a positive number $\gamma \in \mathbb{Q}(\alpha)$ such that $\sigma(\gamma)=-\gamma$, for instance, $\gamma=\alpha-\sigma(\alpha)$ (one may replace $\alpha$ by $-\alpha$, and so, we can suppose $\alpha-\sigma(\alpha)>0)$, then we have $\log (-\sigma(\gamma) / \gamma)=0$, and by (12), we get $\left|\operatorname{Tr}\left(\gamma \omega^{m}\right)\right|_{p} \asymp|m|_{p}$. By Lemma 2.1, there exists a positive integer $A$ such that for every large $m$, the numbers $q=q(m)=A \operatorname{Tr}\left(\gamma \omega^{m}\right)$ are positive integers satisfying (2). For $m=p^{s}$ with $s \in \mathbb{N}$, we get $|m|_{p}=1 / m$, hence $|q|_{p} \asymp 1 / m$. Since $m \asymp \ln q$, we have thus proved that there exists an infinite set of integers $q>1$ satisfying (2) and (3) (which is Theorem 1.1). In this way we obtain integers $q>1$ satisfying (2) and such that $|q|_{p}$ $\asymp 1 / \ln q$.

One can ask whether there exists an infinite set of integers $q>1$ satisfying (2), with

$$
\inf |q|_{p} \ln q=0 .
$$

Given a positive decreasing sequence $\left(\epsilon_{m}\right)$ with $\sum_{m=0}^{+\infty} \epsilon_{m}=+\infty$, a $p$-adic version [4] of Khintchine's Theorem ensures that for almost all $x \in$ $\mathbb{Z}_{p}$, there exist infinitely many positive integers $m$ such that $|x-m|_{p} \leq \epsilon_{m}$. One often considers as reasonable the hypothesis that a given "special" irrational number $x \in \mathbb{Z}_{p}$ satisfies this condition, with $\epsilon_{m}=1 /(m \ln m)$ for $m>1$ (which is false if $x \in \mathbb{Z}_{p} \cap \mathbb{Q}$, since in this case, we have $|x-m|_{p} \gg 1 / m$ for $m$ large). Let us prove that we can choose $\gamma>0$ in $\mathbb{Q}(\alpha)$, with $\left|\frac{\sigma(\gamma)}{\gamma}+1\right|_{p}<|\omega-1|_{p}$, such that $\frac{\log (-\sigma(\gamma) / \gamma)}{\log \omega}$ is an irrational number in $\mathbb{Z}_{p}$. In order to make this obvious, we prove:

Lemma 2.5. There exists $\xi \in \mathbb{Q}(\alpha)$ such that $\xi$ is not a unit, $N_{\mathbb{Q}(\alpha): \mathbb{Q}} \xi=1$, and $|\xi|_{p}=1$.

Proof. The number $\omega$ is a root of the equation $\omega^{2}-S \omega+1=0$, where $S$ is a rational integer, $S=\operatorname{Tr} \omega$. The number $\xi$ must be a root of an equation $\xi^{2}-t \xi+1=0$, where $t$ is a rational number for which there exists a positive 
rational number $\rho$ such that $t^{2}-4=\rho^{2}\left(S^{2}-4\right)$. Such pairs $(t, \rho)$ can be expressed by using a rational parameter $\theta$ :

$$
\begin{gathered}
t=\frac{2\left(S^{2}-4\right) \theta^{2}+2}{\left(S^{2}-4\right) \theta^{2}-1}=2+\frac{4}{\left(S^{2}-4\right) \theta^{2}-1} \\
\rho=\frac{4 \theta}{\left(S^{2}-4\right) \theta^{2}-1} .
\end{gathered}
$$

Let us show that we can choose $\theta \in \mathbb{Q}^{*}$ such that $t \notin \mathbb{Z}$ and $|t|_{p} \leq 1$. It is enough to take $\theta=p$. As we have $S^{2}>4$, hence $S^{2} \geq 9$ and $\left(S^{2}-4\right) p^{2}-1>4, t$ cannot be an integer for this choice of $\theta$. But we have $|t|_{p} \leq 1$, since $\left|\left(S^{2}-4\right) p^{2}-1\right|_{p}=1$. Then there exists a number $\xi \in \mathbb{Q}(\alpha)$ such that $\xi^{2}-t \xi+1=0$, and $\xi$ is neither a rational number, since $\rho>0$, nor an algebraic integer, since $t \notin \mathbb{Z}$. Then we have $N_{\mathbb{Q}(\alpha) / \mathbb{Q}}(\xi)=1$, and $|\xi|_{p}=1$ because either condition $|\xi|_{p}<1$ or $|\xi|_{p}>1$ would imply $|t|_{p}=\left|\xi+\xi^{-1}\right|_{p}>1$.

Replacing $\xi$ by $\xi^{n}$, where $n$ is a suitable positive integer, we thus may find a $\xi$ satisfying Lemma 2.5 , with moreover $|\xi-1|_{p}<|\omega-1|_{p}$. Then we have $|\log \xi|_{p}<|\log \omega|_{p}$. Further let us prove that $\frac{\log \xi}{\log \omega} \in \mathbb{Q}_{p}$. Indeed that is trivial if $\alpha \in \mathbb{Q}_{p}$, since in this case $\xi$ and $\omega$ lie in $\mathbb{Q}_{p}$, hence so do $\log \xi$ and $\log \omega$. If $\mathbb{Q}_{p}(\alpha)$ has degree 2 over $\mathbb{Q}_{p}$, then $\log \xi$ and $\log \omega$ lie in $\mathbb{Q}_{p}(\alpha)$. But $\sigma$ can be extended into a continuous $\mathbb{Q}_{p}$-automorphism of $\mathbb{Q}_{p}(\alpha)$, and we get $\sigma\left(\frac{\log \xi}{\log \omega}\right)=\frac{\log \sigma(\xi)}{\log \sigma(\omega)}=\frac{-\log \xi}{-\log \omega}=\frac{\log \xi}{\log \omega}$, since $\xi \sigma(\xi)=\omega \sigma(\omega)=1$. That proves that $\frac{\log \xi}{\log \omega} \in \mathbb{Q}_{p}$, and since $|\log \xi|_{p}<|\log \omega|_{p}$, we conclude that $\frac{\log \xi}{2 \log \omega} \in \mathbb{Z}_{p}$. Lastly, $\frac{\log \xi}{\log \omega}$ is not a rational number, since $\xi$ is not a unit. Now, by Hilbert's Theorem, there exists $\gamma \in \mathbb{Q}(\alpha)$, with $\gamma>0$, such that $\xi=-\sigma(\gamma) / \gamma$. We thus have found $\gamma>0$ in $\mathbb{Q}(\alpha)$, such that $\left|\frac{\sigma(\gamma)}{\gamma}+1\right|_{p}<p^{-1 /(p-1)}$ and $\frac{\log (-\sigma(\gamma) / \gamma)}{2 \log \omega}$ is an irrational element of $\mathbb{Z}_{p}$. Under the above hypothesis, it would exist infinitely many integers $m>1$ with $\left|\frac{\log (-\sigma(\gamma) / \gamma)}{2 \log \omega}-m\right|_{p} \ll 1 /(m \log m)$, and, by (12), we could obtain an infinite set of integers $q>1, q=A \operatorname{Tr}\left(\gamma \omega^{m}\right)$ where $A$ is a positive integer, satisfying (2) and such that $|q|_{p} \ll \frac{1}{\ln q \ln \ln q}$. In particular, (3') would be satisfied.

\section{Conclusion}

For a sequence $\mathcal{B}$ bounded, the Roth-Ridout Theorem [6] allows us to see that for any irrational algebraic real number $\alpha$, thus in particular for $\alpha$ quadratic, we have:

$$
\inf _{q>0} q^{1+\epsilon}\|q \alpha\||q|_{\mathcal{B}}>0
$$


(see [5]). Of course, our method is far from enabling us to prove that there exists a real constant $\lambda$ such that

$$
\inf _{q>1} q(\ln q)^{\lambda}\|q \alpha\||q|_{\mathcal{B}}>0 .
$$

We can only study the approximations with $q\|q \alpha\| \ll 1$. It seems difficult to study approximations in the "orthogonal direction" $q|q|_{\mathcal{B}} \ll 1$, with for instance, $q=p^{n}$, for a prime number $p$. For such approximations, it is not known whether $\inf _{n \in \mathbb{N}}\left\|p^{n} \alpha\right\|=0$ holds, neither if there exists $\lambda$ such that $\inf _{n>0} n^{\lambda}\left\|p^{n} \alpha\right\|>0$. It is very difficult to obtain more precise results than the Roth-Ridout Theorem (see [1]).

Even for rational approximations satisfying (2), we are not able to prove that the constants $\lambda(\mathcal{S})$ are bounded. This is related to Lemma 2.4. It would be necessary to prove that there exists a real constant $\kappa$ for which this Lemma holds for $x=\omega$ and for any $y \in \mathbb{Q}(\alpha)$ with $|y-1|_{p}<p^{-1 /(p-1)}$ and $N_{\mathbb{Q}(\alpha) / \mathbb{Q}}(y)=1$. There exist many effective estimates of $|k \log x+\ell \log y|_{p}$ (see for instance [2] and [8]), but they do not provide the needed result. It seems difficult to take the particular conditions required into account.

Acknowledgements. The author thanks warmly Michel Waldschmidt for their lighting conversations about linear forms in logarithms, at Graz. The author also thanks the referee.

\section{References}

[1] M. BAuer, M. BennetT, Applications of the hypergeometric method to the generalized Ramanujan-Nagell equation. Ramanujan J. 6 (2002), 209-270.

[2] Y. Bugeaud, M. LAURent, Minoration effective de la distance p-adique entre puissances de nombres algébriques. J. Number Theory 61 (1996), 311-342.

[3] B. DE MATHAN, Linear forms in logarithms and simultaneous Diophantine approximation. (To appear).

[4] B. DE MAthan, Approximations diophantiennes dans un corps local. Bull. Soc. math. France, Mémoire 21 (1970).

[5] B. de Mathan, O. Teulí́, Problèmes diophantiens simultanés. Monatshefte Math. 143 (2004), 229-245.

[6] D. RIDout, Rational approximations to algebraic numbers. Mathematika 4 (1957), 125131.

[7] L. G. PECK, Simultaneous rational approximations to algebraic numbers. Bull. Amer. Math. Soc. 67 (1961), 197-201.

[8] K. YU, p-adic logarithmic forms and group varieties II. Acta Arith. 89 (1999), 337-378.

Bernard DE MATHAN

Université Bordeaux I

UFR Math-Info. Laboratoire A2X

351 cours de la Libération

33405 Talence, France

E-mail : Bernard.de-Mathan@math.u-bordeaux1.fr 